\title{
Regulation of myelin genes implicated in psychiatric disorders by functional activity in axons
}

\author{
Philip R. Lee and R. Douglas Fields* \\ National Institutes of Health, NICHD, Bethesda, MD, USA
}

\section{Edited by:}

Kathleen S. Rockland, RIKEN Brain

Science Institute, Japan

Reviewed by:

NobuakiTamamaki, Kumamoto

University, Japan

Gundela Meyer, Universidad de La Laguna, Spain

*Correspondence:

Dr. R. Douglas Fields, Nervous System Development and Plasticity Section,

National Institutes of Health, NICHD,

Bldg. 35, Room 2A211, MSC 3713, 35

Lincoln Drive, Bethesda, MD 20892

USA.

e-mail: fieldsd@mail.nih.gov
Myelination is a highly dynamic process that continues well into adulthood in humans. Several recent gene expression studies have found abnormal expression of genes involved in myelination in the prefrontal cortex of brains from patients with schizophrenia and other psychiatric illnesses. Defects in myelination could contribute to the pathophysiology of psychiatric illness by impairing information processing as a consequence of altered impulse conduction velocity and synchrony between cortical regions carrying out higher level cognitive functions. Myelination can be altered by impulse activity in axons and by environmental experience. Psychiatric illness is treated by psychotherapy, behavioral modification, and drugs affecting neurotransmission, raising the possibility that myelinating glia may not only contribute to such disorders, but that activity-dependent effects on myelinating glia could provide one of the cellular mechanisms contributing to the therapeutic effects of these treatments. This review examines evidence showing that genes and gene networks important for myelination can be regulated by functional activity in axons.

Keywords: oligodendrocyte, axon, activity, schizophrenia, depression, white matter, ATP, LIF

\section{INTRODUCTION}

The establishment and development of psychiatric disorders are likely to involve aberrant regulation and expression of many genes, together with multiple environmental factors, ultimately leading to illness. In recent years researchers have begun to focus on the potential role of white matter and oligodendrocytes in the pathophysiology of psychiatric disorders (for a recent review see Dwork et al., 2007). Myelination can be viewed as a highly dynamic process which can be altered by impulse activity in axons (Demerens et al., 1996; Stevens et al., 1998) and by environmental factors. It is becoming clear that myelination continues into adulthood and may contribute to plasticity of cognitive function, learning and memory (Fields, 2005, 2008). Perturbations in the molecular processes leading to axon myelination will consequently result in axon dysfunction and abnormal electrical conduction, therefore impairing the transfer of information across brain regions. It is likely that axon health and dysfunction contribute to the pathophysiology of a number of psychiatric disorders, and axon survival is dependent on the close association of axons with myelinating glia (Nave and Trapp, 2008). The guiding hypothesis for this review is that in addition to the well appreciated synaptic dysfunction in psychiatric disorders, oligodendrocytes also play a major role, and that myelination by oligodendrocytes well into adulthood may be regulated by the firing of action potentials in axons. This type of regulation may be analogous to activity-dependent changes in neurons and synaptic connectivity as a consequence of environmental stimuli (for a recent review see Fields et al., 2005).

There are several mechanisms by which oligodendrocytes could sense functional activity in axons (Figure 1). Oligodendrocytes at various stages of development have ion channels, purinergic and other membrane receptors that allow myelinating glia to detect impulse activity through the activity-dependent release of molecules from axons (Figures 1B,D,E). Thus activity-dependent regulation of oligodendrocytes could contribute to cellular mechanisms promoting recovery through environmental interventions and other non-drug treatments of psychiatric illnesses. Drug treatments for neuropsychiatric illnesses may also act in part through effects on myelinating glia. Oligodendrocytes have neurotransmitter receptors for glutamate, serotonin, and dopamine, making it likely that antipsychotic drugs acting through these neurotransmitter systems would also have actions on myelinating glia that may be detrimental or beneficial in psychiatric disorders. Finally, synaptic communication between axons and immature myelinating glia (oligodendrocyte progenitor cells), have been described recently in white matter (Karadottir et al., 2008; Kukley et al., 2007; Lin et al., 2005), providing a rapid means of direct communication between axons and myelinating glia.

Myelination is a complex biological process that involves an intricate regulatory network among many different cell types in the nervous system (Rosenberg et al., 2007). Many of the genes revealed in genomic studies of mental illness that are crucial to the normal functioning of the myelination program and myelinmaintenance are themselves candidates for regulation by electrical activity in axons. Many of these genes relate to oligodendroglia function; however some of these genes are expressed in astrocytes and some in neurons where they may have independent effects or act indirectly on myelinating glia.

\section{TRANSCRIPTIONAL REGULATION OF MYELIN GENES IN OLIGODENDROCYTES}

Regulating transcription of structural components of myelin, such as PLP1, MBP, MAG, MOG, and CNP is clearly critical in the process of oligodendrocyte development and the subsequent correct myelination of specific axons. Several of these major components 


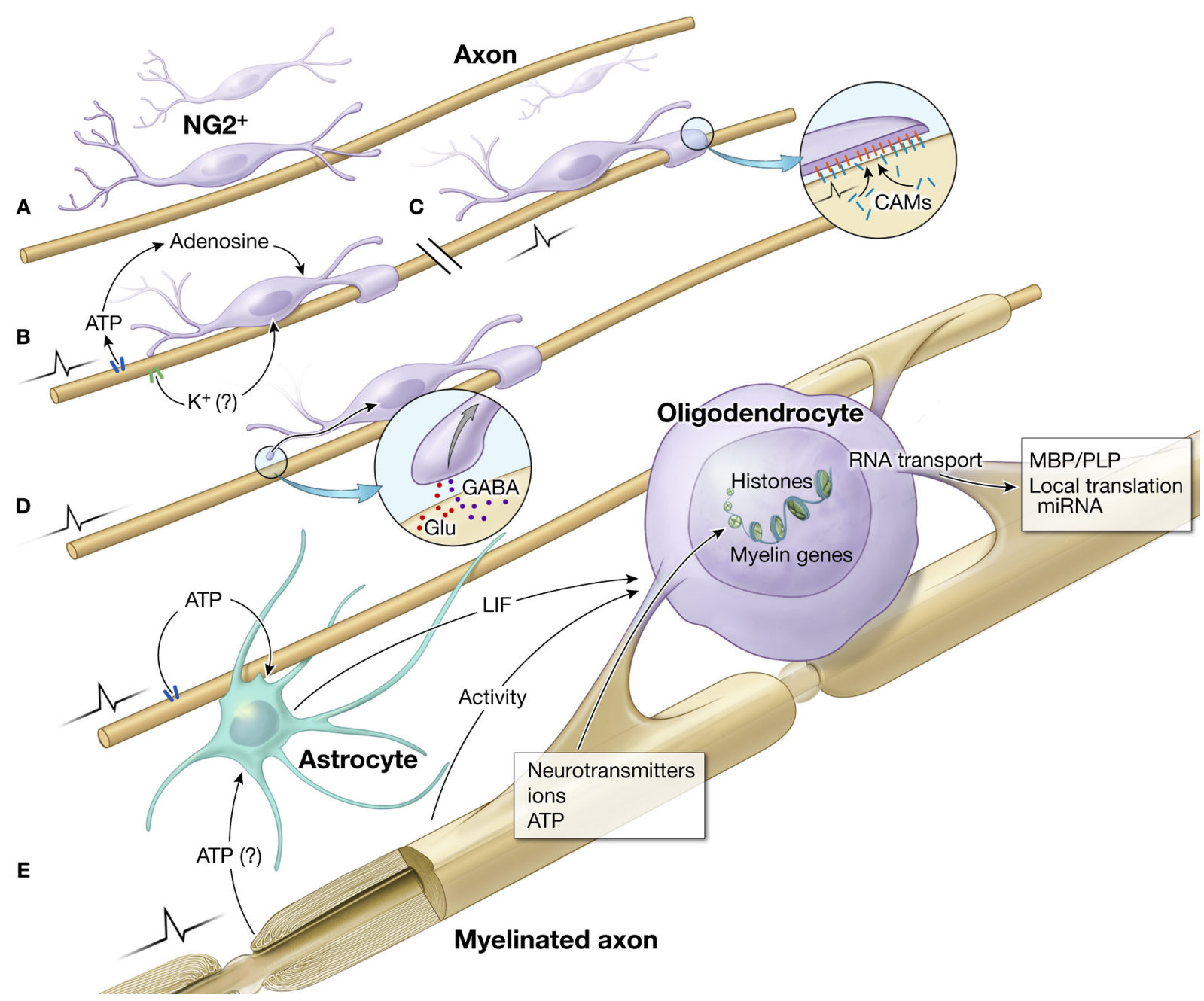

FIGURE 1 | Impulse activity in axons regulates oligodendrocyte development and myelination at several stages and via different signals.

(A) Immature OPCs (NG2+ cells) in white matter on an electrically silent unmyelinated axon. Such cells persist in significant numbers in the adult brain. (B) Electrical activity causes ATP release from axons, which generates adenosine that stimulates differentiation of NG2 cells to a mature oligodendrocyte, and promotes myelination (Stevens et al., 2002). $\mathrm{K}^{+}$is released from electrically active axons. Blocking $\mathrm{K}^{+}$channels in oligodendrocytes in culture has been shown to regulate oligodendrocyte proliferation and lineage progression (Ghiani et al., 1999). (C) Electrical activity can also alter the expression of cell adhesion molecules on the axon that are involved in initiating myelination (Itoh et al., 1995, 1997). This has been shown to regulate myelination by Schwann cells in the PNS, but the same molecule (L1-CAM) is involved in myelination by oligodendrocytes (Barbin et al., 2004). (D) The release of the neurotransmitters Glu (glutamate) or GABA from synapses formed on NG2 cells (Kukley et al., 2007), could provide another mechanism to regulate myelination in response to functional activity. (E) After NG2 cells differentiate into oligodendrocytes, ATP released from axons firing action potentials stimulates the synthesis and release of the cytokine LIF from astrocytes, which promotes myelination (Ishibashi et al., 2006). Myelination during development and postnatally may be regulated by several other unidentified activity-dependent signaling molecules affecting development of oligodendrocytes and myelin formation. Electrical activity in axons, via the release of neurotransmitters, ions and ATP may influence gene expression in oligodendrocytes by histone modification, RNA transport, local translation and regulate mRNA stability and translation by miRNAs. of myelin have been shown to be regulated by action potential firing or by alterations in intracellular calcium or cAMP (Atkins et al., 1997, 1999; Gao et al., 2004; Studzinski et al., 1999); both of these second messengers can be regulated by neural impulse activity. Phosphorylation of MBP is regulated by MAP kinase in response to action potential firing during long-term potentiation (LTP) in the hippocampus (Atkins et al., 1999), a cellular model of memory, and by direct electrical stimulation of white matter in hippocampus. The functional significance of MBP phosphorylation in oligodendrocyte development and myelination is unclear. Additionally the level of
mRNA and protein for MBP and PLP in an oligodendrocyte cell line are sensitive to increased intracellular calcium (Studzinski et al., 1999) and myelin associated glycoprotein (MAG) is sensitive to levels of cAMP (Gao et al., 2004). Therefore several of the major myelin genes can be regulated by electrical activity or mechanisms by which second messenger signal transduction can be modified. Many of the major components of myelin are deregulated in psychiatric disorders, particularly in schizophrenia (Hakak et al., 2001).

In order for myelination to proceed, a complex network of transcriptional repression and activation must be activated (Wegner, 
2008). Several of the transcription factors required for repression and activation of myelin genes have been found to be abnormally expressed in the brains of patients with psychiatric disorders and in particular those with schizophrenia (Katsel et al., 2005).

Of particular interest is the transcription factor SOX10 (Tkachev et al., 2003) which is required for the expression of two of the major components of myelin, the proteins MBP and PLP1 (Stolt et al., 2002). Recently it has been shown that in Schwann cells, the myelinating glia of the peripheral nervous system, Sox10 is a component of a calcium-sensitive transcriptional complex (Kao et al., 2009). Calcium is the primary second messenger communicating action potential firing to intracellular responses (Eshete and Fields, 2001), and signaling to the nucleus to regulate gene transcription (Fields et al., 2005). The Sox10/NFAT complex is critical for Schwann cell development and activates several genes known to be regulators of myelination in the peripheral nervous system, such as KROX20 (Kao et al., 2009). Interestingly the SOX10 gene is located in a major susceptibility locus for schizophrenia and reduced expression of this gene was found to be correlated with an increase in the methylation state of the allele found in schizophrenia patients (Iwamoto et al., 2005).

An increase in the methylation of chromatin is indicative of a transcriptionally inactive state and it will be interesting to see if other transcription factors and genes required for oligodendrocyte function are regulated in this manner. It is well established that epigenetic modification of chromatin structure by DNA methylation is a critical event in the transcriptional regulation of gene expression (Kouzarides, 2007). In neurons DNA methylation can be affected by membrane depolarization and electrical activity in a Gadd45b-dependent manner (Ma et al., 2009). It is unknown if epigenetic regulation in oligodendrocytes can be regulated through a similar activity-dependent mechanism. However, the alteration of chromatin structure by histone deactylases (HDACs) is also thought to play a major role in the repression of myelin gene transcriptional inhibitors such as Tcf4 and Id4 (He et al., 2007a). However repression of the negative regulators of myelin gene transcription is in itself not sufficient to allow immature oligodendrocytes ( $\mathrm{NG}^{+}$ cells) to progress to more mature developmental stage and begin the process of myelination (He et al., 2007b; Figure 2).

External signals generated by electrical activity in axons, such as ATP or glutamate release from axons, can cause changes in intracellular calcium levels in oligodendrocytes and therefore these axonally derived signals may play a role in the epigenetic regulation of the transcriptional apparatus required for lineage progression and myelination by oligodendrocytes and in the process of remyelination (Figure 1E). Clearly a perturbation of this type of epigenetic regulation in psychiatric disorders, perhaps by soluble axon-derived signals such as ATP or glutamate, would provide a link with environmental cues as axon firing patterns reflect environmental stimuli.

\section{REGULATION OF MYELIN GENES BEYOND TRANSCRIPTION}

The complexity of mRNA expression and metabolism and the localization of specific mRNAs to subcellular compartments in oligodendrocytes will all contribute to the eventual pool of mRNA available for the translational machinery. RNA transport, splicing and stability mechanisms are tightly regulated by intracellular signal transduction in many cell types in the nervous system.
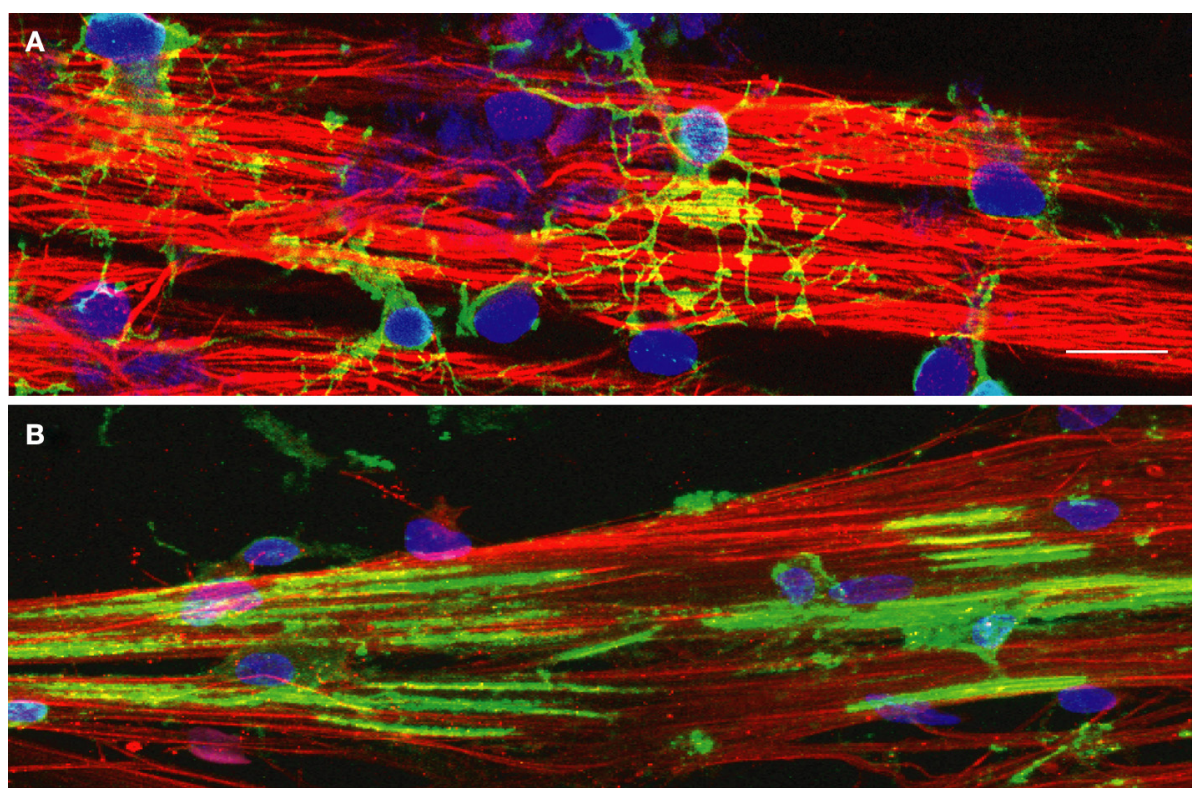

FIGURE 2 | NG2+ cells and MBP+ oligodendrocytes grown on DRG axons in culture. DRG axons grown in culture for 3 weeks were immunostained for neurofilament (red) to identify axons and DAPI (blue) to label cell nuclei. (A) Immature oligodendrocyte progenitor cells (OPCs) were plated onto DRG axons and immunostained for the immature OPC marker NG2 (green) after 2 days in co-culture. $\mathrm{NG}^{+}$positive OPCs have differentiated into multipolar cells with many processes contacting multiple axons in order to initiate the myelination process. (B) OPCs grown in co-culture with DRG axons for 7 days were immunostained with myelin basic protein (MBP), a component of the myelin sheath. Immature OPCs have differentiated into a myelination phenotype with expression of MBP and the formation of multiple segments of compact myelin associated with the axons. Scale bar $=15 \mu \mathrm{m}$. 
Interestingly, many of the myelin genes are alternatively spliced during development and several of these mRNAs are targets for transport by specific RNA binding proteins (McInnes and Lauriat, 2006). Importantly, a broad spectrum of RNA binding proteins has been found to be deregulated in schizophrenia (Katsel et al., 2005), including quaking (QKI) and hnRNAPA2. Deregulation of such a large group of RNA binding proteins will have many downstream consequences for RNA metabolism and localization in oligodendrocytes. For example, Aberg et al. (2006) demonstrated that deregulation of several genes expressed in oligodendrocytes and associated with schizophrenia were associated with alternative expression of QKI splice variants. Therefore disruption in the expression of the QKI gene has downstream consequences for oligodendrocyte development and myelination and this may lead to a predisposition to psychiatric illness.

It is also known that hnRNPA2 is a carrier protein for MBP mRNA in oligodendrocytes (Ainger et al., 1997); it is thought that hnRNPA2-MBP complexes are transported into oligodendrocyte processes on microtubules (Carson and Barbarese, 2005), however the axonal signal for this mechanism is not thought to be a soluble factor released from the axon, but instead a cell adhesion molecule expressed on the axon (White et al., 2008). Nonetheless it is attractive to hypothesize that RNA metabolism beyond transcriptional control may also be subject to regulation by extracellular signaling cues derived from electrically active axons. There is evidence in the literature that in neuronal processes RNA transport is regulated by electrical activity (Willis et al., 2007). A similar type of regulation could in theory control RNA transport in the polarized process of oligodendrocytes in contact with individual axons. This type of regulation may provide precise control, in response to signals derived from electrically active axons, of the available mRNA pool of myelin genes in individual processes of developing and mature oligodendrocytes.

Another potential mechanism by which post-transcriptional regulation of oligodendrocyte-related genes may be accomplished is to regulate mRNA homeostasis by the binding of specific micro RNAs (miRNAs) to myelin gene mRNAs. Micro RNAs are regulators of translation and RNA stability (Kosik and Krichevsky, 2005); this is achieved by miRNA binding to the UTR of target RNAs and directly influencing the amount of mRNA available to the translational machinery. The study of the regulation of miRNAs in the brain is still at a relatively early stage, however the functional targets of several miRNAs have been described in neurons (Fiore et al., 2008) and oligodendrocytes (Lau et al., 2008). Interestingly, a very recent study has suggested the involvement of a miRNA (miR219) in NMDA signaling (Kocerha et al., 2009), whereby blocking NMDA receptor function by regulating a CaMKII subunit that signals downstream of this receptor, a similar effect was seen by treating mice with antipsychotic medication. It is interesting to note that mature oligodendrocytes also express NMDA receptors on myelinating processes (for a recent review of glutamate signaling in white matter see Bakiri et al., 2009) but the role of these receptors is currently unknown. Additionally Beveridge et al. (2008) reported that levels of another miRNA (miR-181b) are elevated in schizophrenia; targets of this miRNA are downregulated in the same brain region as the miRNA is upregulated in. Of the miRNAs known to function in neurons, several have been shown to be regulated by electrical activity in neuronal processes (for example Wayman et al., 2008). It is tempting to speculate that miRNA regulation of translation in oligodendrocytes may operate in a similar manner in response to soluble factors released from electrically active axons, such as BDNF, glutamate, ATP and GABA. Given the very early stage of determining functions for miRNAs in oligodendrocyte biology, further work is required to show the specific miRNAs that may target many of the genes implicated in oligodendrocyte function and are implicated in psychiatric disorders.

\section{REGULATION OF GENES IN ASTROCYTES}

In addition to oligodendrocyte-related genes, other non-neuronal genes such as glial fibrillary acidic protein (GFAP), an intermediate filament protein expressed in astrocytes, have been implicated in psychiatric disorders (Martins-de-Souza et al., 2008; Torrey et al., 2000). Astrocytes can have an important influence on development of oligodendrocytes by secretion of trophic factors and cytokines. GFAP is an integral component of the astrocyte cytoskeleton and altered expression of GFAP can have many effects on astrocyte biology (Haydon, 2001). Therefore GFAP dysregulation and by implication astrocytic dysfunction could have profound effects on axon health, neuro-transmission, and neuron-glia signaling, and perhaps most importantly indirectly in the process of myelination. Additionally, GFAP expression is thought to be regulated by DNA methylation (Song and Ghosh, 2004) and recent evidence suggests that activity in neuronal circuits can regulate the expression of GFAP in hippocampal cell culture (Cohen and Fields, 2008). Therefore extracellular factors generated by neuronal activity have been shown to regulate GFAP expression and astrocyte differentiation and this could occur through the regulation of chromatin structure. Astrocytes have also been shown to have a direct role in regulating myelination and oligodendcrocyte development. Studies in cell culture have shown that astrocytes promote myelination in response to electrical stimulation of axons via the release of a cytokine Leukemia Inhibitory Factor (LIF), in response to ATP released from electrically active axons (Ishibashi et al., 2006). Taken together it is becoming clear that astrocyte function, which can be regulated by neuronal activity, can have profound effects on the myelination process and may contribute to disease progression in psychiatric disorders (Figure 1E).

\section{REGULATION OF GENES IN AXONS}

Another mechanism by which electrical activity could influence gene expression in oligodendrocytes is by the expression of cell surface signaling molecules such as specific cell adhesion receptors in axons (Figure 1C). This general type of mechanism has already been shown to regulate myelination in the peripheral nervous system (Stevens et al., 1998). In these studies, different cell adhesion molecules on axons, NCAM, N-Cadherin, and L1-CAM were regulated by specific frequencies of electrical stimulation (Itoh et al., 1995, 1997). L1-CAM is required for initiation of myelination by Schwann cells (Wood et al., 1990) and downregulating the L1 gene by electrical stimulation at the appropriate frequency inhibited myelin formation in cell culture (Stevens et al., 1998). L1-CAM is also required for myelination by oligodendrocytes (Barbin et al., 2004), suggesting that specific patterns of impulse activity could affect myelination in the brain and spinal cord. 
The expression of specific proteins and protein complexes at sites of axon-glia contact could provide a direct link between axon signaling and the regulation of gene expression in oligodendrocytes (Figure 1C). In support of this idea it has been demonstrated that extracellular stimuli can regulate the localization of specific mRNAs in axons (Willis et al., 2007), and it has been demonstrated that the expression of cell surface receptors can be modulated by electrical activity in axons (Itoh et al., 1995). Therefore, taken together, regulation of myelination by an activity-dependent signaling cascade, originating in axons, may allow direct coupling of neuronal activity and oligodendrocyte intracellular signaling.

In an interesting recent study by White et al. (2008) regulation of localized MBP translation in oligodendrocytes has been shown to be dependent upon the neuronal adhesion molecule L1 binding to oligodendrocytes resulting in Fyn-kinase activation and translation of MBP mRNA in oligodendrocyte processes in contact with the axon. This study potentially links electrical activity in axons via L1 expression activation of Fyn-kinase, resulting in increased translation of MBP in oligodendrocyte processes. Perturbation of any part of this signaling mechanism could result in defective myelin. This type of axon-glia interaction may be a common regulatory mechanism by which myelin deposition is targeted to the correct axon site in response to the expression of a cell surface receptor by impulse activity in the axon.

\section{ACTIVITY DEPENDENT REGULATION OF AXON-DERIVED DIFFUSIBLE MOLECULES}

Importantly, it has been demonstrated that diffusible molecules released from axons firing action potentials can be detected by myelinating glia, with subsequent control of glial development and myelination. Cell culture studies have shown that action potentials induced by electrical stimulation release ATP from axons, which activates $\mathrm{P} 2$ receptors on myelinating glia of the peripheral nervous system (Schwann cells). This regulates proliferation and differentiation of Schwann cells in accordance with functional activity in axons, by stimulating calcium influx, activation of CaMKII, MAPK, and the transcription factors c-fos, Krox24, and CREB (Stevens and Fields, 2000). P2 receptors are also present on oligodendrocyte at early stages of development (Fields and Burnstock, 2006). Adenosine, derived from ATP released from electrically active axons, acts on immature oligodendrocytes to promote differentiation and myelination (Stevens et al., 2002; Figure 1B). Other molecules released by electrically active axons that could in theory influence myelinating glia include potassium $\left(\mathrm{K}^{+}\right)$and neurotransmitters. Several studies have demonstrated that blockade of $\mathrm{K}^{+}$channels or membrane depolarization with veratridine inhibits oligodendrocyte cell proliferation and differentiation (Ghiani et al., 1999). Growth factors, such as BDNF, which are known regulators of oligodendrocyte differentiation (Van't Veer et al., 2009), can be secreted or regulated in an activity-dependent manner and by environmental experience, providing another potential general mechanism for activity-dependent regulation of myelination. Interactions between BDNF and serotonin in mood disorders have been reported (Martinowich and Lu, 2008), and the BDNF gene has been associated with increased risk for a number of neuropsychiatric disorders (Martinowich et al., 2007).

\section{REGULATION OF GENE EXPRESSION AND PHARMACOLOGICAL INTERVENTION IN PSYCHIATRIC DISORDERS}

Drug treatments for psychiatric disorders that correct deregulation of genes involved in myelination and oligodendrocyte dysfunction are an appealing possibility. In this regard pharmacological regulation of the activity of specific histone deacetylases (HDACs) is an interesting avenue of investigation, although therapeutic intervention of the modulation of HDAC activity in mouse models of demyelination has shown mixed results. Therefore it may be important to identify cell-type specific regulators of chromatin structure as it relates to oligodendrocyte function, thus targeting any drug treatments more specifically to limit off-target effects on other cell populations in the brain. Regulation of gene expression by medication beyond transcriptional regulation may provide a more specific mechanism to target myelin genes in oligodendrocytes. In this regard a very interesting piece of preliminary data comes from the study by Aberg et al. (2006) who show that the level of QKI mRNA can be influenced by medication used to treat schizophrenic patients. Modulation of QKI levels would have effects on the mRNA levels and cellular localization of many of the myelin genes and therefore oligodendrocyte function in the process of myelination. This research is at an early stage; however it opens up new routes that may be used to correct the defects seen in myelin gene expression in oligodendrocytes.

A recent study (Roy et al., 2007) implicates erb-signaling in oligodendrocytes in the functioning of dopaminergic neurons. Erb4 and its ligand neuregulin have been linked genetically to several psychiatric disorders (Corfas et al., 2004). This finding is perhaps of great clinical relevance as many antipsychotic medications work through modulation of dopamine. It is currently unclear exactly how dopamine function is disrupted by oligodendrocyte dysfunction; however this finding provides yet another link between oligodendrocyte biology and the correct functioning of neurons.

The extent to which dopamine levels could lead to mental illness in part through effects on myelinating glia, and whether antipsychotic treatments have therapeutic action in part through effects on oligodendrocytes are two intriguing questions of current investigation. There is evidence that dopamine can influence oligodendrocyte development and function. Both D2 and D3 dopamine receptors are expressed in oligodendrocytes or oligodendrocyte progenitors (Bongarzone et al., 1998; Howard et al., 1998). Quetiapine, a D2 receptor agonist used as an antipsychotic drug, increases synthesis of myelin basic protein and facilitates myelination in rat embryonic cortical cultures (Xiao et al., 2008). The D2/D3 agonist quinpirole also increases the number of oligodendrocyte progenitor cells (OPCs) and it decreases the number of mature oligodendrocytes in primary cell culture. Dopamine also can be toxic to oligodendrocyte progenitors by inducing superoxide generation and lowering glutathione levels (Hemdan and Almazan, 2008). Agonists for dopamine D2 and D3 receptors have been shown to provide significant protection of oligodendrocytes against oxidative injury (Rosin et al., 2005). On the contrary, haloperidol, a typical antipsychotic drug blocking D2 activity reduces myelin proteins in mice treated for 30 days (Narayan et al., 2007). 
Schizophrenia and depression can also involve imbalances in the neurotransmitter serotonin, and several drug treatments act through regulating serotonin levels, for example the serotonin reuptake inhibitor fluoxetine (PROZAC). Serotonin receptors are expressed in Schwann cells (Gaietta et al., 2003), the myelinating glia of the PNS, and the human polymavirus, JC virus, which causes multifocal leukoencaphalopathy, binds the 5HT2a serotonin receptor (Elphick et al., 2004) on oligodendrocyte progenitor cells (Schaumburg et al., 2008). The serotonin antagonists, metoclopramidine, chlorpromazine, clozapine, and serotonin itself all significantly inhibit viral infection (Elphick et al., 2004), which indicates that medications affecting serotonin levels could influence oligodendrocytes. Indeed, the antidepressant drug, fluoxetine, increases cell proliferation of precursors in cell culture that can give rise to astrocytes, neurons, or oligodendrocytes (Zusso et al., 2004). Serotonin injection into the CNS of dogs has long been known to cause severe demyelination (Saakov et al., 1977), and serotonin reduces the number of oligodendrocytes in adult mouse brain (Moller, 2007).

Other neurotransmitters can regulate different steps of oligodendrogliogenesis through such ion channels and receptors as the delayed $\mathrm{K}^{+}$rectifier, the AMPA/kainate, dopamine or muscarinic receptors (review see Belachew et al., 1999). This suggests the possibility for activity-dependent regulation of oligodendrocyte differentiation and myelination, and raises the possibility of medications acting on neurotransmitters or the excitation of specific circuits could influence oligodendrocytes.

\section{CONCLUSIONS AND FUTURE WORK}

Evidence from multiple areas of research including human brain imaging studies and large scale mRNA profiling analyses strongly indicate that defects in myelin and abnormal expression of myelin genes, and their regulators, are common in many psychiatric disorders. It is plausible that perturbations in axon conductance brought about by defects in myelin are responsible for many of the cognitive impairments seen in psychiatric disorders. It is also clear that oligodendrocyte development and myelination and the process of remyelination involve a complex network of intracellular modification and extracellular signaling cues. It is apparent that activity in axons can not only regulate gene expression in the axon, but may also in turn regulate gene expression in oligodendrocytes. Therefore the identification of signaling cues generated by axons firing action potentials, which may be responsible for regulating gene expression in oligodendrocytes, is likely to be an important area for future research.

The situation is complex as both positive and negative regulators of oligodendrocyte function will be involved, and growth

\section{REFERENCES}

Aberg, K., Saetre, P., Lindholm, E., Ekholm, B., Pettersson, U., Adolfsson, R., and Jazin, E. (2006). Human QKI, a new candidate gene for schizophrenia involved in myelination. Am. J. Med. Genet. B Neuropsychiatr. Genet. 141B, 84-90.

Ainger,K.,Avossa,D., Diana,A.S., Barry, C., Barbarese, E., and Carson, J. H. (1997).
Transport and localization elements in myelin basic protein mRNA. J. Cell Biol. 138, 1077-1087.

Atkins, C. M., Chen, S. J., Klann, E., and Sweatt, J. D. (1997). Increased phosphorylation of myelin basic protein during hippocampal long-term potentiation. J. Neurochem. 68, 1960-1967.

Atkins, C. M., Yon, M., Groome, N. P., and Sweatt, J. D. (1999). Regulation of

factors, cytokines, and neurotransmitters can have pronounced dose-dependent effects that may stimulate or inhibit oligodendrocyte development. Research thus far has identified three molecular mechanisms regulating myelination by action potentials in axons: (1) adenosine derived from ATP released from electrically active axons stimulates differentiation of OPCs (Stevens et al., 2002); (2) after oligodendrocytes mature, ATP released from axons acts on astrocytes to stimulate the synthesis and release of LIF which promotes myelination (Ishibashi et al., 2006); (3) action potentials at appropriate frequencies down regulate the cell adhesion molecule L1-CAM to inhibit myelination (Stevens et al., 1998).

Many other soluble factors released from axons firing action potentials, in addition to ATP and adenosine, are potential candidates for regulating myelination, such as GABA, glutamate, and nitric oxide; growth factors, such as BDNF, or enzymes that are able to modify protein complexes expressed on the surface of oligodendrocyte processes, are also likely to be involved in activitydependent myelination. Changes in specific complexes of proteins expressed on axons in response to firing patterns, notably cell adhesion molecules and other membrane receptors, is another general mechanism regulating oligodendrocyte development and myelination to neural impulse activity. It is likely that a combination of all these intercellular communication pathways, and multiple cell types, are at work regulating myelinating glia in the mammalian brain in accordance with functional activity in individual axons in neural circuits mediating cognitive function.

Researchers are only beginning to develop an understanding of how electrical activity can regulate myelin formation. Even less is known about axon-derived soluble factors and their possible role in regulating other aspects of oligodendrocyte biology, such as cell polarity and vesicle trafficking. It is still unclear if the myelin abnormalities seen in psychiatric disorders are the cause of axon dysfunction or a consequence of it, but there is strong evidence to suggest both processes are at work. Effects of drugs that are used in treating psychiatric illnesses on myelinating glia, suggest that some of the beneficial action could be mediated through effects on oligodendrocytes, and conversely, that the cellular changes in white matter seen in schizophrenia, chronic depression, and other psychiatric illnesses could be induced in part by the treatments. Only a much clearer understanding of the complex relationship between myelinating oligodendrocytes and axons (Figures 1 and 2) will allow novel therapies to be developed in the treatment of myelin dysfunction and psychiatric disease.

\section{ACKNOWLEDGMENT}

This work was supported by NICHD funds for intramural research.

myelin basic protein phosphorylation by mitogen-activated protein kinase during increased action potential firing in the hippocampus. J. Neurochem. 73, 1090-1097.

Bakiri, Y., Burzomato, V., Frugier, G. Hamilton, N. B., Karadottir, R., and Attwell, D. (2009). Glutamatergic signaling in the brain's white matter. Neuroscience 158, 266-274.
Barbin, G., Aigrot, M. S., Charles, P., Foucher,A., Grumet, M., Schachner, M., Zalc, B., and Lubetzki, C. (2004).Axonal cell-adhesion molecule L1 in CNS myelination. Neuron Glia Biol. 1, 65-72.

Belachew, S., Rogister, B., Rigo, J. M., Malgrange, B., and Moonen, G. (1999). Neurotransmitter-mediated regulation of CNS myelination: a review. Acta Neurol. Belg. 99, 21-31. 
Beveridge, N. J., Tooney, P.A., Carroll, A. P., Gardiner, E., Bowden, N., Scott, R. J., Tran, N., Dedova, I., and Cairns, M. J. (2008). Dysregulation of miRNA $181 \mathrm{~b}$ in the temporal cortex in schizophrenia. Hum. Mol. Genet. 17, 1156-1168.

Bongarzone, E. R., Howard, S. G., Schonmann, V., and Campagnoni, A.T. (1998). Identification of the dopamine D3 receptor in oligodendrocyte precursors: potential role in regulating differentiation and myelin formation. J. Neurosci. 18, 5344-5353.

Carson, J. H., and Barbarese, E. (2005). Systems analysis of RNA trafficking in neural cells. Biol. Cell. 97, 51-62.

Cohen, J. E., and Fields, R. D. (2008). Activity-dependent neuron-glial signaling by ATP and leukemia-inhibitory factor promotes hippocampal glial cell development. Neuron Glia Biol. 4, 43-55.

Corfas, G., Roy, K., and Buxbaum, J. D. (2004). Neuregulin 1-erbB signaling and the molecular/cellular basis of schizophrenia. Nat. Neurosci. 7, 575-580.

Demerens, C., Stankoff, B., Logak, M., Anglade, P., Allinquant, B., Couraud, F., Zalc, B., and Lubetzki, C. (1996). Induction of myelination in the central nervous system by electrical activity. Proc. Natl. Acad. Sci. U.S.A. 93, 9887-9892.

Dwork, A. J., Mancevski, B., and Rosoklija, G. (2007). White matter and cognitive function in schizophrenia. Int. J. Neuropsychopharmacol. 10, 513-536.

Elphick, G. F., Querbes, W., Jordan, J. A., Gee, G. V., Eash, S., Manley, K., Dugan, A., Stanifer, M., Bhatnagar, A., Kroeze, W. K., Roth, B. L., and Atwood, W. J. (2004). The human polyomavirus, JCV, uses serotonin receptors to infect cells. Science 306, 1380-1383.

Eshete, F., and Fields, R. D. (2001). Spike frequency decoding and autonomous activation of $\mathrm{Ca}^{2+}$-calmodulindependent protein kinase II in dorsal root ganglion neurons. J. Neurosci. 21, 6694-6705.

Fields, R. D. (2005). Myelination: an overlooked mechanism of synaptic plasticity? Neuroscientist 11, 528-531.

Fields, R. D. (2008). White matter in learning, cognition and psychiatric disorders. Trends Neurosci. 31, 361-370.

Fields, R. D., and Burnstock, G. (2006). Purinergic signalling in neuron-glia interactions. Nat. Rev. Neurosci. 7 , 423-436.

Fields, R. D., Lee, P. R., and Cohen, J. E. (2005). Temporal integration of intracellular $\mathrm{Ca}^{2+}$ signaling networks in regulating gene expression by action potentials. Cell Calcium 37, 433-442.
Fiore, R., Siegel, G., and Schratt, G. (2008). MicroRNA function in neuronal development, plasticity and disease. Biochim. Biophys. Acta 1779, 471-478.

Gaietta, G. M., Yoder, E. J., Deerinck, T., Kinder, K., Hanono, A., Han, A., Wu, C., and Ellisman, M. H. (2003). 5-HT2a receptors in rat sciatic nerves and Schwann cell cultures. J. Neurocytol. 32, 373-380.

Gao, Y., Deng, K., Hou, J., Bryson, J. B., Barco, A., Nikulina, E., Spencer, T., Mellado, W., Kandel, E. R., and Filbin, M. T. (2004). Activated CREB is sufficient to overcome inhibitors in myelin and promote spinal axon regeneration in vivo. Neuron 44 609-621.

Ghiani, C. A., Yuan, X., Eisen, A. M., Knutson, P. L., DePinho, R. A., McBain, C. J., and Gallo, V. (1999). Voltage-activated $\mathrm{K}^{+}$channels and membrane depolarization regulate accumulation of the cyclin-dependent kinase inhibitors p27(Kip1) and p21(CIP1) in glial progenitor cells. J. Neurosci. 19, 5380-5392.

Hakak, Y., Walker,J. R., Li, C., Wong, W. H., Davis, K. L., Buxbaum, J. D., Haroutunian, V., and Fienberg, A. A. (2001). Genome-wide expression analysis reveals dysregulation of myelination-related genes in chronic schizophrenia. Proc. Natl. Acad. Sci. U.S.A. 98, 4746-4751.

Haydon, P. G. (2001). GLIA: listening and talking to the synapse. Nat. Rev. Neurosci. 2, 185-193.

He, Y., Dupree, J., Wang, J., Sandoval, J., Li, J., Liu, H., Shi, Y., Nave, K. A., and Casaccia-Bonnefil, P. (2007a) The transcription factor Yin Yang 1 is essential for oligodendrocyte progenitor differentiation. Neuron 55, 217-230.

He, Y., Sandoval, J., and CasacciaBonnefil, P. (2007b) Events at the transition between cell cycle exit and oligodendrocyte progenitor differentiation: the role of HDAC and YY1. Neuron Glia Biol. 3, 221-231.

Hemdan, S., and Almazan, G. (2008). Dopamine-induced toxicity is synergistically potentiated by simultaneous HSP-90 and Akt inhibition in oligodendrocyte progenitors. J. Neurochem. 105, 1223-1234.

Howard, S., Landry, C., Fisher, R., Bezouglaia, O., Handley, V., and Campagnoni, A. (1998). Postnatal localization and morphogenesis of cells expressing the dopaminergic D2 receptor gene in rat brain: expression in non-neuronal cells. J. Comp. Neurol. 391, 87-98.

Ishibashi, T., Dakin, K. A., Stevens, B., Lee, P. R., Kozlov, S. V., Stewart, C. L., and Fields, R. D. (2006). Astrocytes promote myelination in response to electrical impulses. Neuron 49 , 823-832.

Itoh, K., Ozaki, M., Stevens, B., and Fields, R. D. (1997). Activitydependent regulation of $\mathrm{N}$-cadherin in DRG neurons: differential regulation of $\mathrm{N}$-cadherin, NCAM, and L1 by distinct patterns of action potentials. J. Neurobiol. 33, 735-748.

Itoh, K., Stevens, B., Schachner, M., and Fields, R. D. (1995). Regulated expression of the neural cell adhesion molecule L1 by specific patterns of neural impulses. Science 270, 1369-1372.

Iwamoto, K., Bundo, M., Yamada, K. Takao, H., Iwayama-Shigeno, Y., Yoshikawa, T., and Kato, T. (2005) DNA methylation status of SOX10 correlates with its downregulation and oligodendrocyte dysfunction in schizophrenia. J. Neurosci. 25 , 5376-5381.

Kao, S. C., Wu, H., Xie, J., Chang, C. P., Ranish, J. A., Graef, I. A., and Crabtree, G. R. (2009). Calcineurin/ NFAT signaling is required for neuregulin-regulated Schwann cell differentiation. Science 323, 651-654.

Karadottir, R., Hamilton, N. B., Bakiri, Y., and Attwell, D. (2008). Spiking and nonspiking classes of oligodendrocyte precursor glia in CNS white matter. Nat. Neurosci. 11, 450-456.

Katsel, P., Davis, K. L., Gorman, J. M., and Haroutunian, V. (2005). Variations in differential gene expression patterns across multiple brain regions in schizophrenia. Schizophr. Res. 77 241-252.

Kocerha, J., Faghihi, M. A., LopezToledano, M. A., Huang, J., Ramsey, A. J., Caron, M. G., Sales, N., Willoughby,D.,Elmen,J.,Hansen, H. F., Orum, H., Kauppinen, S., Kenny, P. J. and Wahlestedt, C. (2009). MicroRNA219 modulates NMDA receptor-mediated neurobehavioral dysfunction. Proc. Natl. Acad. Sci. U.S.A. 106, 3507-3512.

Kosik, K. S., and Krichevsky, A. M. (2005). The elegance of the MicroRNAs: a neuronal perspective. Neuron 47, 779-782.

Kouzarides, T. (2007). Chromatin modifications and their function. Cell 128, 693-705.

Kukley, M., Capetillo-Zarate, E., and Dietrich, D. (2007). Vesicular glutamate release from axons in white matter. Nat. Neurosci. 10, 311-320.

Lau, P., Verrier,J.D., Nielsen, J.A., Johnson, K. R., Notterpek, L., and Hudson, L. D. (2008). Identification of dynamically regulated microRNA and mRNA networks in developing oligodendrocytes. J. Neurosci. 28, 11720-11730.

Lin, S. C., Huck, J. H., Roberts, J. D., Macklin, W. B., Somogyi, P., and
Bergles, D. E. (2005). Climbing fiber innervation of NG2-expressing glia in the mammalian cerebellum. Neuron 46, 773-785.

Ma, D. K., Jang, M. H., Guo, J. U., Kitabatake, Y., Chang, M. L., PowAnpongkul, N., Flavell, R. A., Lu, B., Ming, G. L., and Song, H. (2009). Neuronal activity-induced Gadd45b promotes epigenetic DNA demethylation and adult neurogenesis. Science 323, 1074-1077.

Martinowich, K., and Lu, B. (2008). Interaction between BDNF and serotonin: role in mood disorders. Neuropsychopharmacology 33, 73-83.

Martinowich, K., Manji, H., and Lu, B. (2007). New insights into BDNF function in depression and anxiety. Nat. Neurosci. 10, 1089-1093.

Martins-de-Souza, D., Gattaz, W.F. Schmitt, A., Maccarrone, G., HunyadiGulyas, E., Eberlin, M. N., Souza, G. H. Marangoni, S., Novello, J. C., Turck, C. W., and Dias-Neto, E. (2008). Proteomic analysis of dorsolateral prefrontal cortex indicates the involvement of cytoskeleton, oligodendrocyte, energy metabolism and new potential markers in schizophrenia. J. Psychiatr. Res. [Epub ahead of print].

McInnes, L. A., and Lauriat, T. L. (2006). RNA metabolism and dysmyelination in schizophrenia. Neurosci. Biobehav. Rev. 30, 551-561.

Moller, H.J. (2007). Clinical evaluation of negative symptoms in schizophrenia. Eur. Psychiatry 22, 380-386.

Narayan, S., Kass, K.E., and Thomas, E. A. (2007).Chronic haloperidol treatment results in a decrease in the expression of myelin/oligodendrocyte-related genes in the mouse brain. J. Neurosci. Res. 85, 757-765.

Nave, K. A., and Trapp, B. D. (2008). Axonglial signaling and the glial support of axon function. Ann. Rev. Neurosci. 31, 535-561.

Rosenberg, S. S., Powell, B. L., and Chan, J. R. (2007). Receiving mixed signals: uncoupling oligodendrocyte differentiation and myelination. Cell. Mol. Life Sci. 64, 3059-3068.

Rosin, C., Colombo, S., Calver, A. A., Bates, T. E., and Skaper, S. D. (2005). Dopamine D2 and D3 receptor agonists limit oligodendrocyte injury caused by glutamate oxidative stress and oxygen/glucose deprivation. Glia 52, 336-343.

Roy, K., Murtie, J. C., El-Khodor, B. F., Edgar, N., Sardi, S. P., Hooks, B. M., Benoit-Marand, M., Chen, C., Moore,H., O’Donnell, P., Brunner, D., and Corfas, G. (2007). Loss of erbB signaling in oligodendrocytes alters myelin and dopaminergic function, a potential 
mechanism for neuropsychiatric disorders. Proc. Natl. Acad. Sci. U.S.A. 104, 8131-8136.

Saakov, B. A., Khoruzhaia, T. A., and Bardakhch'ian, E. A. (1977). Ultrastructural mechanisms of serotonin demyelination. Biull. Eksp. Biol. Med. 83, 606-610.

Schaumburg, C., O’Hara, B. A., Lane, T. E., and Atwood, W. J. (2008). Human embryonic stem cell-derived oligodendrocyte progenitor cells express the serotonin receptor and are susceptible to JC virus infection. J. Virol. 82, 8896-8899.

Song, M. R., and Ghosh, A. (2004). FGF2induced chromatin remodeling regulates CNTF-mediated gene expression and astrocyte differentiation. Nat. Neurosci. 7, 229-235.

Stevens, B., and Fields, R. D. (2000). Response of Schwann cells to action potentials in development. Science 287, 2267-2271.

Stevens, B., Porta, S., Haak, L. L., Gallo, V., and Fields, R. D. (2002). Adenosine: a neuron-glial transmitter promoting myelination in the CNS in response to action potentials. Neuron 36 , 855-868.

Stevens, B., Tanner, S., and Fields, R. D. (1998). Control of myelination by specific patterns of neural impulses. $J$. Neurosci. 18, 9303-9311.
Stolt, C. C., Rehberg, S., Ader, M., Lommes, P., Riethmacher, D., Schachner, M., Bartsch, U., and Wegner, M. (2002). Terminal differentiation of myelin-forming oligodendrocytes depends on the transcription factor Sox 10. Genes Dev. 16, 165-170.

Studzinski, D. M., Callahan, R. E., and Benjamins, J. A. (1999). Increased intracellular calcium alters myelin gene expression in the N20.1 oligodendroglial cell line. J. Neurosci. Res. 57, 633-642.

Tkachev, D., Mimmack, M. L., Ryan, M. M., Wayland, M., Freeman, T., Jones, P. B., Starkey, M., Webster, M. J., Yolken, R. H., and Bahn, S. (2003). Oligodendrocyte dysfunction in schizophrenia and bipolar disorder. Lancet 362, 798-805.

Torrey, E. F., Webster, M., Knable, M., Johnston, N., and Yolken, R. H. (2000). The stanley foundation brain collection and neuropathology consortium. Schizophr. Res. 44, 151-155.

Van't Veer, A., Du, Y., Fischer, T. Z., Boetig, D. R., Wood, M. R., and Dreyfus, C. F. (2009). Brain-derived neurotrophic factor effects on oligodendrocyte progenitors of the basal forebrain are mediated through trkB and the MAP kinase pathway. J. Neurosci. Res. 87, 69-78.
Wayman, G. A., Davare, M., Ando, H., Fortin, D., Varlamova, O., Cheng, H. Y., Marks, D., Obrietan, K., Soderling, T. R., Goodman, R.H., and Impey, S. (2008). An activity-regulated microRNA controls dendritic plasticity by down-regulating p250GAP. Proc. Natl. Acad. Sci. U.S.A. 105 9093-9098.

Wegner, M. (2008). A matter of identity: transcriptional control in oligodendrocytes. J. Mol. Neurosci. 35, 3-12.

White, R., Gonsior, C., KramerAlbers, E. M.,Stohr, N.,Huttelmaier, S., and Trotter, J. (2008). Activation of oligodendroglial Fyn kinase enhances translation of mRNAs transported in hnRNP A2-dependent RNA granules. J. Cell Biol. 181, 579-586.

Willis, D. E., van Niekerk, E. A., Sasaki, Y., Mesngon, M., Merianda, T. T., Williams, G. G., Kendall, M. Smith, D. S., Bassell, G. J., and Twiss, J. L. (2007). Extracellular stimuli specifically regulate localized levels of individual neuronal mRNAs. J. Cell Biol. 178, 965-980.

Wood, P. M., Schachner, M., and Bunge, R. P. (1990). Inhibition of Schwann cell myelination in vitro by antibody to the $\mathrm{L} 1$ adhesion molecule. J. Neurosci. 10, 3635-3645.

Xiao, L., Xu, H., Zhang, Y., Wei, Z., He, J., Jiang, W., Li, X., Dyck, L. E.,
Devon, R. M., Deng, Y., and Li, X. M. (2008). Quetiapine facilitates oligodendrocyte development and prevents mice from myelin breakdown and behavioral changes. Mol. Psychiatry 13, 697-708.

Zusso, M., Debetto, P., Guidolin, D., and Giusti, P. (2004). Cerebellar granular cell cultures as an in vitro model for antidepressant drug-induced neurogenesis. Crit. Rev. Neurobiol. 16, 59-65.

Conflict of Interest Statement: The authors declare that the research was conducted in the absence of any commercial or financial relationships that could be construed as a potential conflict of interest.

Received: 08 May 2009; paper pending published: 18 May 2009; accepted: 19 May 2009; published online: 01 June 2009.

Citation: Lee PR and Fields RD (2009) Regulation of myelin genes implicated in psychiatric disorders by functional activity in axons. Front. Neuroanat. (2009) 3:4. doi:10.3389/neuro.05.004.2009

Copyright (C) 2009 Lee and Fields. This is an open-access article subject to an exclusive license agreement between the authors and the Frontiers Research Foundation, which permits unrestricted use, distribution, and reproduction in any medium, provided the original authors and source are credited. 